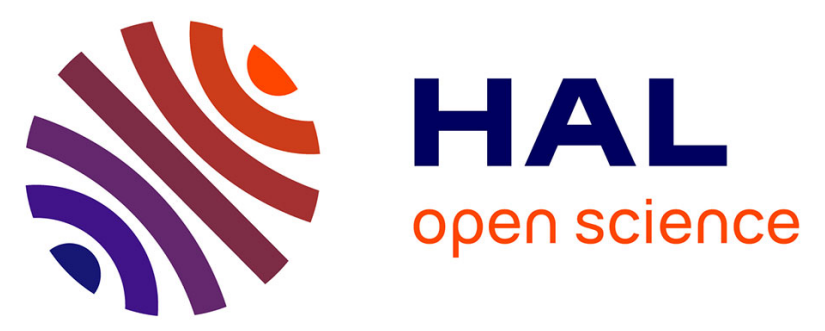

\title{
Two-photon optical imaging, spectral and fluorescence lifetime analysis to discriminate urothelial carcinoma grades
}

\author{
B. Pradère, F. Poulon, E. Comperat, I. Lucas, D. Bazin, S. Doizi, O. \\ Cussenot, O. Traxer, D. Abi Haidar
}

\section{To cite this version:}

B. Pradère, F. Poulon, E. Comperat, I. Lucas, D. Bazin, et al.. Two-photon optical imaging, spectral and fluorescence lifetime analysis to discriminate urothelial carcinoma grades. Journal of Biophotonics, 2018, 11 (11), pp.e201800065. 10.1002/jbio.201800065 . hal-01822699

\section{HAL Id: hal-01822699 \\ https://hal.sorbonne-universite.fr/hal-01822699}

Submitted on 25 Jun 2018

HAL is a multi-disciplinary open access archive for the deposit and dissemination of scientific research documents, whether they are published or not. The documents may come from teaching and research institutions in France or abroad, or from public or private research centers.
L'archive ouverte pluridisciplinaire HAL, est destinée au dépôt et à la diffusion de documents scientifiques de niveau recherche, publiés ou non, émanant des établissements d'enseignement et de recherche français ou étrangers, des laboratoires publics ou privés. 


\title{
Two-photon optical imaging, spectral and fluorescence lifetime analysis to discriminate urothelial carcinoma grades
}

\author{
Authors: B. Pradère ${ }^{1+}$ F. Poulon ${ }^{2+}$, E. Compérat ${ }^{3}$, I. Lucas ${ }^{4}$, D. Bazin ${ }^{5}$, S. Doizi ${ }^{1}$, O. \\ Cussenot $^{1}$, O. Traxer $^{1}$ and D. Abi Haidar, ${ }^{2,6, *}$ \\ Affiliations: \\ 1Department of Urology, Hôpital Tenon, HUEP, AP-HP, Sorbonne University, Paris, France \\ 2IMNC Laboratory, UMR 8165-CNRS/ IN2P3, Paris-Saclay university, 91405 Orsay, France. \\ 3 Department of Pathology, Hôpital Tenon, HUEP, AP-HP, Sorbonne University, Paris, France \\ 4UPMC Univ Paris 06, Sorbonne Universités, UMR 8235, LISE, Paris, F-75252, France \\ 5 Laboratoire de Physique des Solides, CNRS UMR 8502, Université Paris Sud XI, Orsay, France; CNRS, Laboratoire de Chimie de la \\ Matière Condensée de Paris, UPMC, Collège de France, Paris, France \\ 6 Paris Diderot University, Sorbonne Paris Cité, F-75013, Paris, France. \\ +these authors contributed equally to this work
}

\section{* Corresponding author :}

Darine Abi Haidar

IMNC/Université Paris Sud - Bat. 440 - 15 rue Georges Clémenceau 91405 Orsay Cedex, France

Tel : +331691556 29, abihaidar@,imnc.in2p3.fr

Keywords: Bladder tumor, two-photon imaging, endomicroscopy, spectroscopy, fluorescence lifetime imaging

\begin{abstract}
:
In the framework of urologic oncology, mini-invasive procedures have increased in the last few decades particularly for urothelial carcinoma. One of the essential elements in the management of this disease is still the diagnosis, which strongly influences the choice of treatment. The histopathologic evaluation of the tumor grade is a keystone of diagnosis, and tumor characterization is not possible with just a macroscopic evaluation. Even today intraoperative evaluation remains difficult despite the emergence of new technologies which use exogenous fluorophore. This study assessed an optical multimodal technique based on endogenous fluorescence, combining qualitative and quantitative analysis, for the diagnostic of urothelial carcinoma. It was found that the combination of two photon fluorescence, second harmonic generation microscopy, spectral analysis and fluorescence lifetime
\end{abstract}


imaging were all able to discriminate tumor from healthy tissue, and to determine the grade of tumors. Spectral analysis of fluorescence intensity and the redox ratio used as quantitative evaluations showed statistical differences between low grade and high grade tumors. These results showed that multimodal optical analysis is a promising technology for the development of an optical fiber setup designed for an intraoperative diagnosis of urothelial carcinoma in the area of endourology.

\section{Introduction}

With more than 79,000 new cases and a projection of 16,390 deaths in 2017 in the US[1], bladder cancer is one of the deadliest cancer worldwide. For urologists, bladder cancer (BC) and upper urinary tract transitional cell carcinoma (UUT-TCC) are principal issues in urologic oncology. It is therefore critical to diagnose these tumors during endoscopy procedures (cystoscopy or ureteroscopy depending of the tumor localization) in order to confirm lesions macroscopically and to collect a tissue sample to confirm histopathologic characteristics. For specific cases of urothelial carcinoma (BC and UUTTCC), determination of the histopathological grade of urothelial tumors is one of the keystones in the oncological management. The tumor grade impacts the treatment strategy at various levels, from the type of surgery, to the role of chemotherapy, to the chronological steps in management. In the case of a high grade tumor a total ablation of the organ (radical surgery) may be necessary whereas conservative treatment can be a valid option for low grade tumors associated with bladder cancer and upper urinary tract transitional cell carcinoma (UUT-TCC) [2], [3]. A critical step is proper resection of lesions due to a high risk of recurrence[4] particularly for low grade urothelial carcinoma. This is achievable if both lesion detection and diagnosis are optimal. In this framework, peri-operative screening of tumor characteristics could be a valuable support to the surgeon to adapt the surgery and to plan postoperative cares.

With the development of minimally invasive surgery, the use of miniaturized technology has become a standard procedure, especially for UUT-TCC and BC. For UUT-TCC, flexible ureteroscopy with laser photovaporization allows a reduction in both surgical morbidity and renal function impairment [5], 
[6]. New technologies have been developed to help the surgeon with intraoperative discrimination of urothelial carcinoma[7], [8]. Enhanced imaging techniques such as Photodynamic diagnosis (PDD) with 5-ALA[9], Narrow Band Imaging system(NBI)[10], real time optical coherence tomography (OCT)[11] and confocal laser endomicroscopy (CLE)[12] are now proposed for diagnosis. However, all of these technologies have limitations including low specificity (NBI), or difficulty of interpretation by the doctors (OCT, CLE), compared to the gold standard histological diagnosis, due to the lack of multimodality and database images.

After two decades of becoming increasingly popular, two photon microscopy (TPM) has now become an important technique for biological tissue imaging. The development of miniaturized high-numerical aperture optics[13], lasers with optimized pulse delivery [14], and miniaturized scanning system[15], [16] has opened the way for clinical applications. Nonlinear microscopy, as classical confocal imaging, can provide an analysis of tissue using only endogenous labels, and thus eliminates the need for exogenous fluorescence labeling. Second Harmonic Generation (SHG) can access characteristics of tissue structure which have a non-centrosymmetric geometry, such as extra-cellular matrix, muscle fibers, walls of blood vessels, while two photon endogenous fluorescence (TPEF) measurements can detect and show the presence of endogenous fluorophores associated with cellular metabolic activity (reduced nicotinamide adenine dinucleotide: NADH and flavin adenine dinucleotide: FAD).

TPM offers several advantages such as intrinsic sectioning, lower photobleaching, lower and localized phototoxicity, higher penetration depth into the tissues, and an absence of overlap between excitation and emission [17]-[20]. Above all, it provides a different endogenous contrast analysis compared to standard microscopy and confocal endomicroscopy. Although the heterogeneity of tissue structure can lead to complex signal interpretation, endogenous fluorescence analysis of urothelial tissue could be a promising way to avoid metabolic alteration related to external markers[21], [22]. The multimodal technique is an improved alternative to the unimodal one since it highlights tumors characteristics more specifically and precisely.

Nevertheless, non-linear flexible endoscopy imaging development remains a challenge. Although some modalities such as SHG have already been evaluated in-vivo[23] and multiphoton microscopy 
evaluated on urothelial and prostate samples[24], [25], only one study so far has explored the potential of multimodal optical analysis in the area of uro-oncology[26].

This preliminary study was conducted to establish the efficiency of a multimodal imaging process based on endogenous fluorescence analysis to discriminate grades of urothelial tumours as done in histopathologic grading. For that reason, we conducted a qualitative and quantitative study, using different contrasts to define optical markers of tumours grade. This study show that optical imaging and analysis could provide reproducible and reliable clinical results.

To the best of our knowledge, this preliminary study assesses for the first time the ability of multimodal optical analyses to identify pathological features and histopathological grades associated with urothelial carcinoma, in a manner consistent with classical histopathological evaluation.

\section{Material and Methods}

\subsection{Sample management}

This prospective monocentric study had included a collection of samples from patients suspected to have an urothelial carcinoma at fibroscopy or on imagery. The institutional review board (IRB) of the Tenon Hospital center - University Pierre-Marie Curie approved the study (IRB-00003835), all the following methods were performed in accordance with the relevant guidelines and regulations issue in this protocol and informed consent were signed by the patients.

After endoscopic biopsy or resection, urothelial samples were fixed in a formalin zinc solution. Immediately after, the samples were transferred on a specific nonlinear multimodal set-up to perform the acquisitions. After the multimodal optical analysis, the samples were conditioned and analyzed by the pathologist. Detailed clinical data including age at diagnosis, past medical history including urothelial carcinoma and previous treatment were recorded, such as histopathological data.

2.2 Confocal, two-photon microscopy and fluorescence lifetime imaging on a microscopic multimodal setup 
A Mai Tai DeepSee Ti:sapphire laser with automated dispersion compensation (Spectra-Physics, Santa Clara, USA) was used as the source for a TCS SP8 MP microscope (Leica Microsystems, Wetzlar, Germany) to perform two-photon microscopy. The set-up allows us to record imaging, emission spectra and lifetime fluorescence. The laser cavity had over $2.4 \mathrm{~W}$ of average power at 800 $\mathrm{nm}$ and was tunable from $690 \mathrm{~nm}$ to $1040 \mathrm{~nm}$. Two supersensitive Leica hybrid nondescanned (HyD NDD) detectors recorded the faintest structures from deep-tissue sections. For two-photon imaging experiments, fluorescence was collected after the microscope objective via a dichroic beamsplitter, transparent to wavelengths greater than $680 \mathrm{~nm}$. The collected visible spectrum was split by another dichroic cube (FF495-Di03-25 $\times 36$, Semrock, New York, USA) into two channels toward the HyD NDD detectors. This configuration minimized loss in the fluorescence collection. The presence of two additional filters (FF01-448/20-25 and FF01-520/35-25, Semrock, New York, USA) on the dichroic cube set the detection band, respectively one to collect Second Harmonic Generation(SHG) signal and the other one to collect the endogeneous fluorescence.

A hybrid detector was used to perform the spectral acquisition. A prism dispersed the fluorescence and the spectral detected band was selected with a specific motorized split mirror placed before the hybrid detector. The spectral resolution was $10 \mathrm{~nm}$, covering the range from $380 \mathrm{~nm}$ to $780 \mathrm{~nm}$. Spectral acquisition and single-photon counting technology fluorescence lifetime imaging were couples by a unique system which integrated hardware and software (PicoQuant GmbH, Berlin, Germany) with the high-end confocal system Leica TCS SP8 MP.

A spectral mosaic was acquired on a $3 \times 3$ images area selected with the microscope software: a spectral measurement was made for each image of the mosaic then the software merged the information to give a mean spectrum of the mosaic. For fluorescence lifetime imaging measurements, a mosaic of 9 images per sample were analyzed using appropriate excitation wavelengths. The image size was $512 \times$ 512 pixels, at $100 \mathrm{~Hz}$ and represented the average of 10 frames.

Four different optical signals on the region of interest (ROI) were recorded with our multimodal setup: 1) one and two-photon spectral analysis, 2) two-photon fluorescence lifetime imaging measurement, 
3) SHG imaging, and 4) fluorescence imaging under one- and two-photon excitation.

\subsection{Histopathology analysis}

All the samples were analyzed by the same senior uropathologist using hematoxylin-eosin-saffran (HE) staining without prior knowledge of the two-photon analysis status. Results were given according to the WHO 2016 classification for urothelial carcinoma[27]. Correlation between point-topoints optical indexes, imaging abnormalities and pathological examination of biopsy samples in each particular tumor type and grade was performed. All samples were categorized and classified as healthy urothelium or high grade or low grade tumor by the pathologist.

\subsection{Data Analysis}

\subsubsection{Spectral analysis}

Five circular Regions of interest (ROI) of $200 \mu \mathrm{m}$ diameter were placed on each spectral mosaic stack at the $870 \mathrm{~nm}$ excitation wavelength. A spectrum was generated for each ROI using Image $J$ to calculate the mean intensity of each image of the stack. This spectrum was then fitted using a Matlab script developed in the lab and previously used in rat and human brain tumors[28], [29]. The script was readapted to urothelial tissue, two components where identify in the emitted response: the nicotinamide adenine dinucleotide $(\mathrm{NADH})$ and the flavin adenines dinucleotide (FAD). Indeed, the absence of oxygen or a need to increase glucose catabolism cause an increase in NADH fluorescence. Hence, the redox ratio is already well-known to be associated with malignant transformation[30]-[32] Different excitation wavelengths were used. For each excitation wavelength a fit was applied to extract the contribution of the different molecules. Figures 1.a.b. show the response of endogenous molecules using two different excitation wavelengths. As shown, $810 \mathrm{~nm}$ excitation wavelength don't give access to the SHG. Exciting with $870 \mathrm{~nm}$ could be reasonable to collect the metabolic molecules as well as SHG. Consequently, to avoid experimental bias and propose the fastest method to analyze tissue only the $870 \mathrm{~nm}$ wavelength was used in all our analysis. We extracted from this fit the maximum intensity at the max emission wavelength of each component ( $\left.\mathrm{I}_{\max -\mathrm{NADH}}, \mathrm{I}_{\max -\mathrm{FAD}}\right)$ to then calculate a redox ratio, see Figure 1.c. 

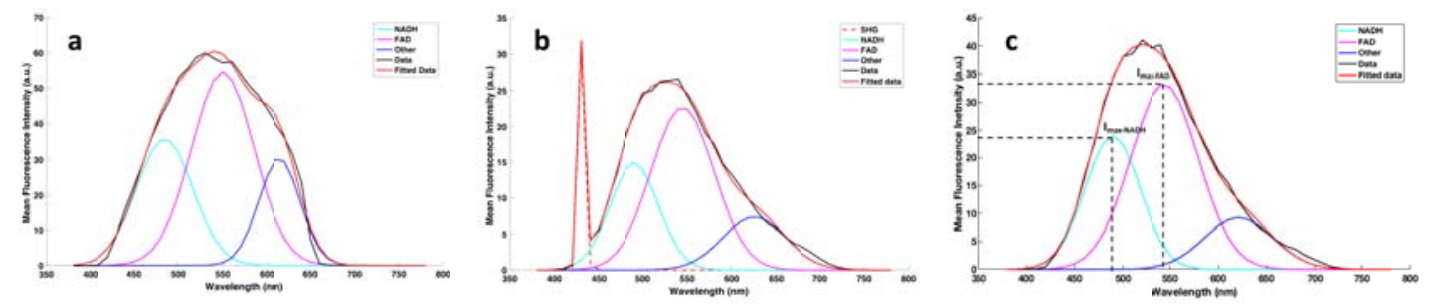

Figure 1. Spectral fluorescence response emitted by the tissue using $810 \mathrm{~nm}$ (a) and $870 \mathrm{~nm}$ (b) excitation wavelengths. The redox ratio was done under 870nm excitation wavelength, using the NADH and FAD fitted intensity (c).

The redox ratio was then calculated from this value with the most used formula in the literature:

$$
\text { Redox }=\frac{\mathrm{I}_{\max -F A D}-\mathrm{I}_{\max -N A D H}}{\mathrm{I}_{\max -F A D}+\mathrm{I}_{\max -N A D H}}[26]
$$

\subsubsection{Lifetime}

The data were collected using the external hybrid dedicated to the fluorescence signal with the filter centered at $520 \pm 35 \mathrm{~nm}$ and analyzed via the software SymphoTime (PicoQuant, GmbH, Berlin, Germany). On each fluorescence lifetime mosaic of nine images, 5 ROI of a $200 \mu \mathrm{m}$ diameter were selected on the cells structures and fitted by bi-exponential decay to extract the fluorescence lifetime. The criteria for an acceptable fit were: 1) a $\chi^{2}$ value less than 1.0 and 2) residuals randomly distributed around 0 within the interval +4 and -4 . The average amplitude lifetime is extracted from fit of each ROI using the following equation:

$$
\text { 2.4.3 Statistical analysis } \tau_{\text {avg }}=\frac{\sum_{i} a_{i} \tau_{i}}{\sum_{i} a_{i}}
$$

The Chi2 test (or Fisher test) were used to compared qualitative variables, the Student t-test were used for quantitative variables. Redox ratio and average fluorescence lifetime results were evaluated using t-test. A probability value $(\mathrm{p})<0.05$ was considered statistically significant. All statistical analyses were performed using R software. 


\section{Results}

\subsection{Samples characteristics:}

In our study, fourteen patients were enrolled prospectively, including sixteen samples of normal and tumoral urothelium. Among them, fifteen samples were of bladder origin and one from the upperurinary tract. Amongst them, ten represented urothelial carcinoma (five reported as low grade tumors and five as high grade tumors). Six samples of non tumoral urothelium were also examined.

\subsection{Correlation between two-photon fluorescence images and Hematoxylin and Eosin staining}

To highlight the performances of non-linear microscopy in tissue diagnosis, we compared our images with the histological analysis, considered as gold standard. Results are shown in Figure 2.

Three major urothelial aspects were identified by fluorescence and compared with the histopathological morphology. First a healthy layer of urothelial cells, second low grade tumor architecture displaying exophytic growth with a fibrovascular core and increased layers of urothelial cells without major atypia or necrosis, and third an invasive high grade tumor, with major atypia and also with exophytic features and a fibrovascular core, typical for pTa tumours (pathologically staged non invasive papillary carcinoma). Moreover, the SHG signal gave us access to the architecture and characteristics of the stroma and the lamina propria and could also be compared with the histological features.

The TPF images were collected through the whole sample, from the epithelial surface to the deepest part, mostly to the detrusor muscle. After the determination of the grade by the multimodal optical analysis, the histopathological images (see Figure 2) were chosen by the pathologist for a better correlation to the TPF images, in order to highlight the specific structures.

Normal urothelium (Figure2; 2a,2d) showed multilayered cells without disorganized architecture, the cells were identify in histological images by dark violet spots and in TPEF images by dark hole in the homogeneous red fluorescent signal, highlighting easily the organization of the layers. There was no 
atypia, no disorganistation and no mitosis. Umbrella cells were present on the surface, no necrotic features were detected.

Low grade tumor (Figure2; 2b, 2e) was recognized by the presence of increased number of urothelial cell layers, an architecture which is still recognizable, no cytonuclear atypia, no mitosis. The fibrovascular core is thin, and was highlight in histological images by pink filament and in two-photon images by the thin green, SHG response, no invasion of the underlying tissue.

High grade tumor (Figure 1; 2c, 2f) is identifiable due to papillary features, the urothelium displays a loss of polarity, cytonuclear atypia can be observed with a modify architecture at the nuclear and epithelial level, this is highlighted in two-photon images by a very irregular form of nuclei of cells, mitosis exist and the fibrovascular core is slightly thickened, the SHG is very difficult to distinguish from the fluorescence, very thin green fiber at the bottom of the image 2.f.
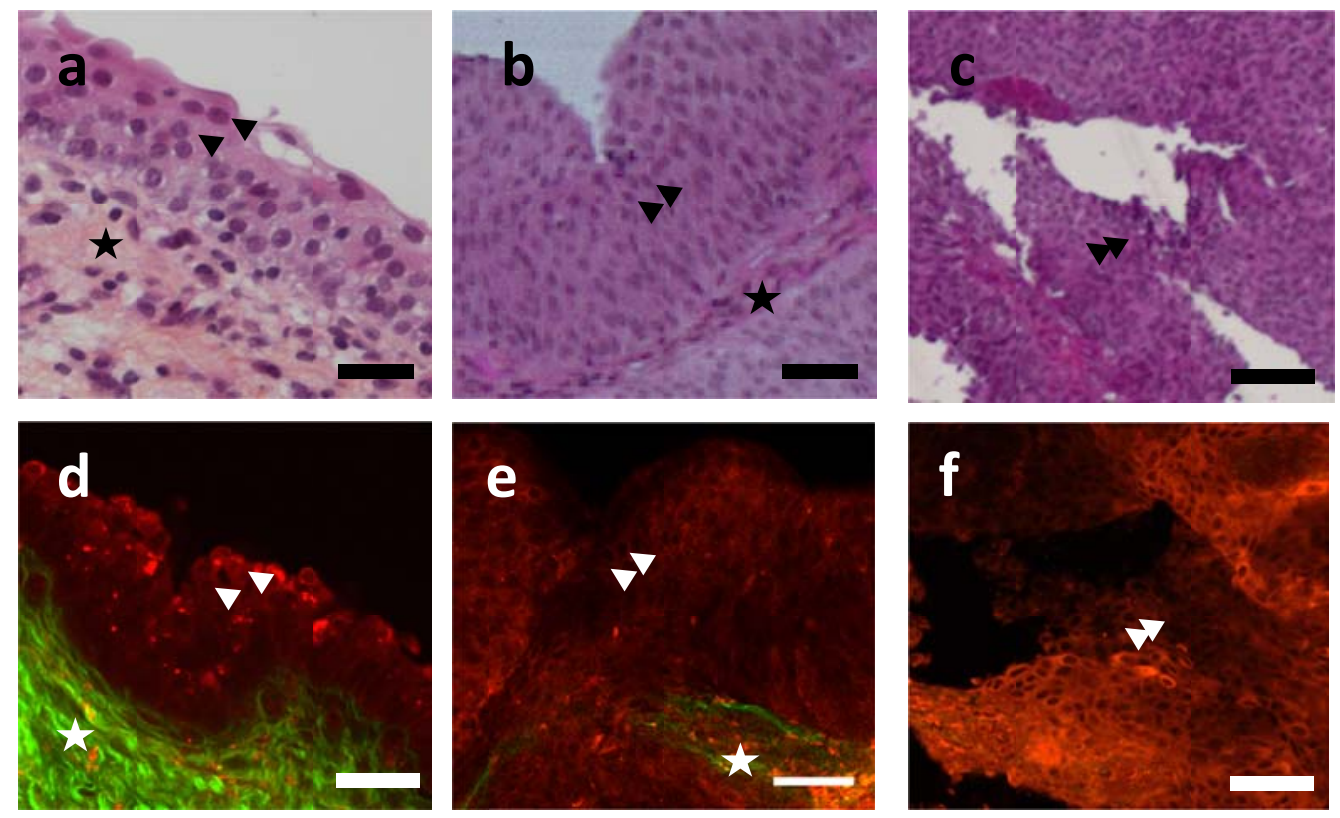

Figure 2. Comparison between Hematoxylin - Eosin Saffran staining (a,b,c) with two-photon fluorescence images at $870 \mathrm{~nm}$ excitation wavelength, red color for the fluorescence signal and green color for the SHG signal $(\mathrm{d}, \mathrm{e}, \mathrm{f})$ of three tissue types : healthy $(\mathrm{a}, \mathrm{d})$, low grade tumor $(\mathrm{b}, \mathrm{e})$ and high grade tumor $(\mathrm{c}, \mathrm{f})$. Scale: $100 \mu \mathrm{m}$. star: Fibrovascular cores, arrow: Urothelial cells.

\subsection{Quantitative evaluation by spectral analysis and fluorescence lifetime imaging}

Once we identified in our images healthy and tumoral urothelium, we performed a quantitative analysis by recording the spectral response and fluorescence lifetime imaging. 
A specific wavelength of $870 \mathrm{~nm}$ has been chosen for the spectral analysis. This wavelength was chosen after an excitation-emission spectral analysis. We excited the same tissue using all excitation range of the laser from 690 to $1040 \mathrm{~nm}$. The maximum of emission fluorescence was recorded using two hybrid detectors over the excitation range (Figure 3).

The Hybrid1 tracks the SHG and Hybrid2 the emitted endogenous fluorescence. The $870 \mathrm{~nm}$ excitation wavelength was chosen to maximize both signal.

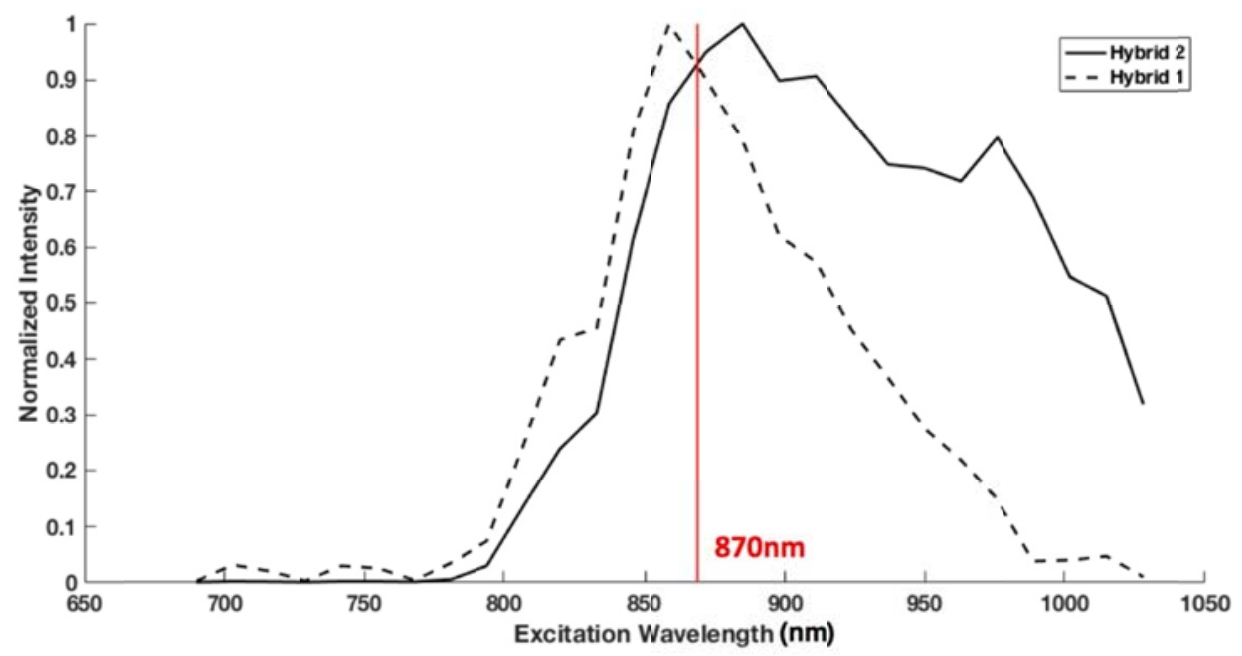

Figure 3. Different fluorescent emission with different excitation wavelength (690-1030nm) in hybrid NDD detector.

The spectral results are shown on the Figure 4. We compared the intensity level of the emitted spectra in the Fig.4a. The higher the grade is, the lower is the fluorescence signal. Healthy urothelium has the higher fluorescence intensity.

Then we were able to calculate the redox ratio for each tissue group. The results are presented in Fig.4.b.c. The redox ratio was significantly higher in the healthy urothelium compared with tumors samples $(\mathrm{p}<0.001)$. Moreover, it was able to discriminate low grade from high grade tumors. The low grade has a significantly higher redox ratio $(\mathrm{p}=0.002)$ than high grade tumor. 

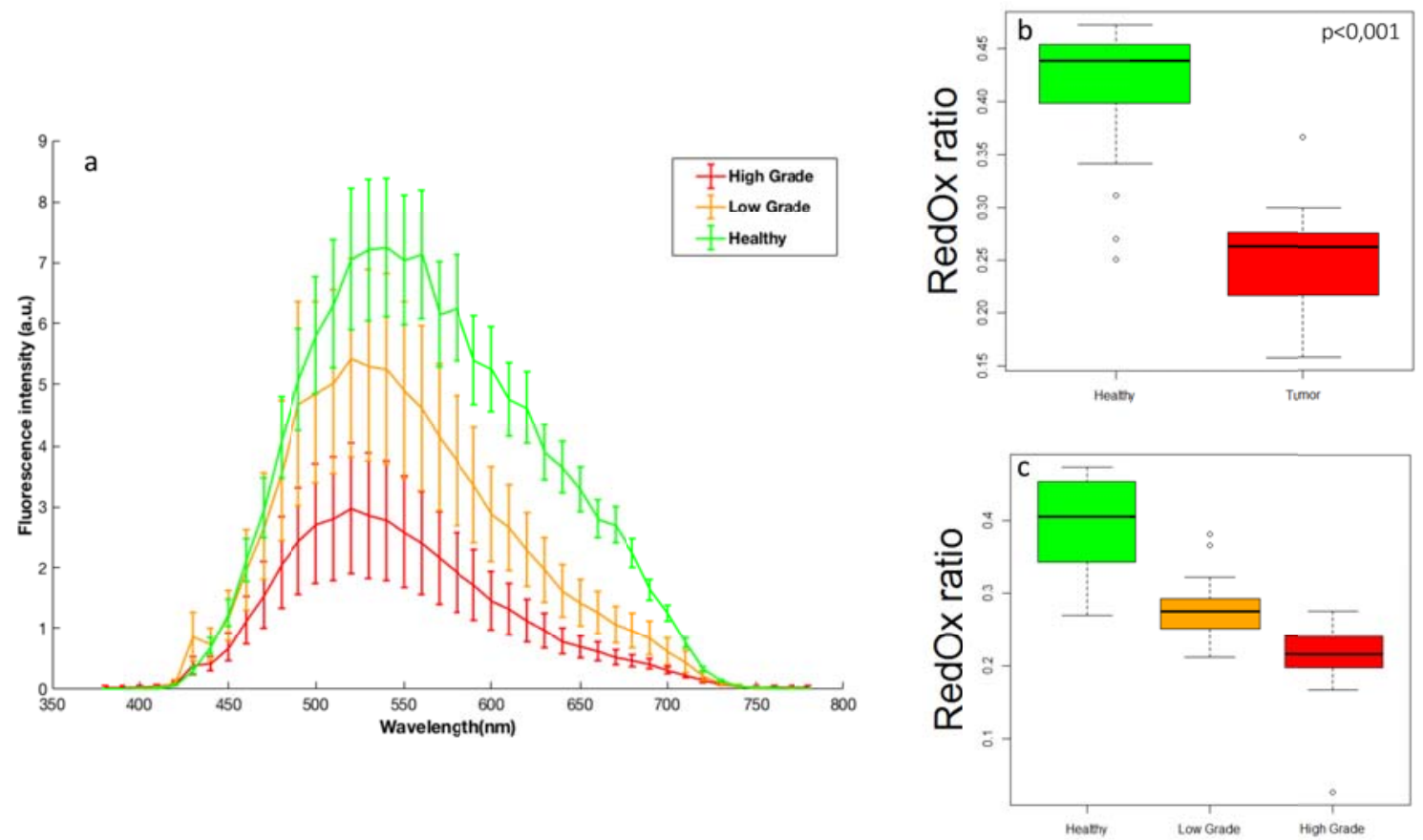

Figure 4. Spectral analysis of urothelial tissue at an excitation of $870 \mathrm{~nm}$. (a) Comparison of the mean spectra in normal urothelium, low and high grade tumor ( 5 samples of each). (b) Comparison of the redox ratio in fifteen region of interest (ROI) in each type between healthy urothelium and tumors. (c) Comparison of redox ratio including low and high grade urothelial carcinoma.

To have a reliable answer and more discriminating indicator, the fluorescence lifetime was also measured. The different results are summarized in Figure 5. We were able to identify the cells structure in the FLIM images and to compare the different grades. This analysis also allowed us to discriminate the different origins. The average lifetime in low grade tumor appeared shorter on our images and in the histogram, Fig.5.a.b.c.

To have a numerical discriminating indicator, fluorescence decay was fitted by a bi-exponential fit in fifteen ROIs and the amplitude lifetime was extracted. Fig.5.d. presents the result, and shows significant differences between healthy, low grade and high grade urothelial carcinoma: healthy vs. low grade $(\mathrm{p}=0.002)$, healthy vs. high grade $(\mathrm{p}<0.001)$ and high grade $v$ s. low grade $(\mathrm{p}<0.001)$. Tumor tissue have a different metabolic equilibrium than normal tissue, in which the co-enzyme $\mathrm{NADH}$ and FAD are implied. The variation in the fluorescence lifetime translate this different metabolic state. 

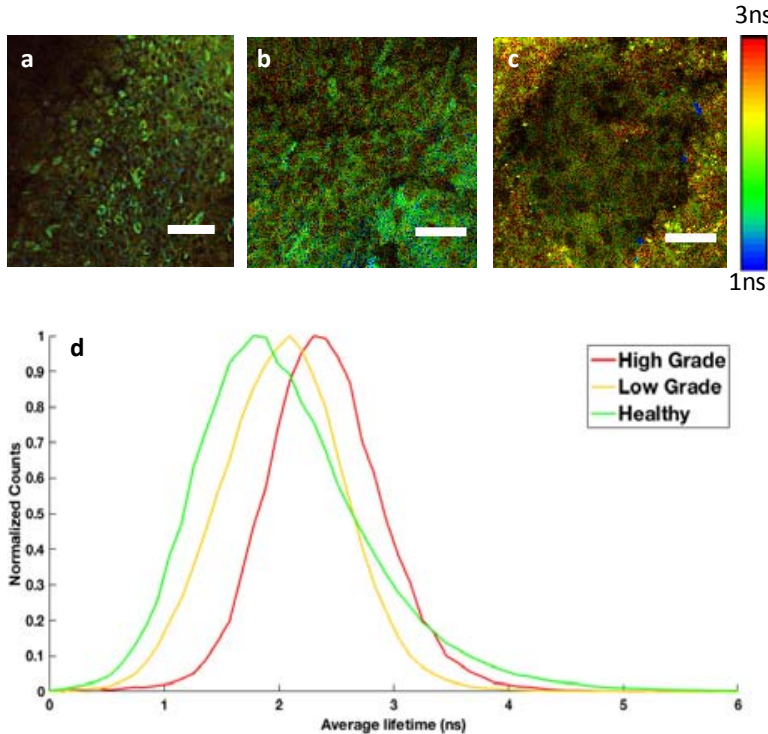

Figure 5. Fluorescence Lifetime analysis at 870nm excitation wavelength. FLIM images of (a) healthy, (b) low and (c)high grade tumor cells, (d) respective average lifetime histogram of each image.(e) Variation of mean amplitude lifetime between healthy urothelium, low grade and high grade tumor compared in fifteen ROIs in each type of tissue.

\section{Discussion and conclusion}

The multimodal optical analysis of urothelial samples from bladder and urinary upper tract has proven its capacity to characterize the urothelium and discriminate healthy tissue, low grade and high grade tumor. This characterization was achieved by a qualitative and a quantitative analysis. These preliminary results are promising for the development of a peri-operative diagnostic tool.

Multiphoton microscopy is a rising technology in the field of tissue analysis. Its intrinsic characteristics make it an increasingly studied technique even in urology[25], [33]. Indeed, in urology, some studies have already shown a good correlation between multi-photonic microscopy and histopathologic imaging. Yadav et al. have demonstrated the discrimination of periprostatic neural tissue on a rat model with using $780 \mathrm{~nm}$ as excitation wavelength excitation[25]. For urothelial carcinoma, Jain et al.[33] have assessed the diagnostic potential of this technique in identifying and differentiating benign from malignant flat bladder lesion, especially carcinoma in situ (CIS). These studies have shown a good correlation between multi-photonic microscopy and histopathology. However, none of them have performed a quantitative analysis such as spectral analysis or FLIM analysis. Multimodality as quantitative and qualitative analysis is important for reliable and precise 
response. Moreover, Jain et al. have focused only on the capacity to detect CIS but have not sought to determine papillary tumors characteristics especially the grade. As PDD and NBI are performant tools for the CIS diagnosis, it is more interesting to develop a specific peri-operative tool able to help determine the histologic grade in order to improve oncologic management.

When multiphoton microscopy is used alone, it is still limited by the difficulty of easily recognizing abnormal aspects such as intranuclear modifications. In our study, multiphonic imaging also lead us to recognize and discriminate structures as seen in histopathology. In particular, it was possible to discern healthy urothelium from low and high grade tumors. It is very interesting and promising that optical imaging could be used for live peri-operative histology. It offers high resolved, rapid images comparable to histological images. But because of the learning curve for the tissue recognition and potential intraoperative variability, imaging alone is not sufficient for a reproducible diagnostic tool. This is why, multimodality by adding quantitative analysis to qualitative makes major contribution to clinical applicability in endo-urology.

Our study has shown that fluorescent spectral analysis can discriminate among different types of urothelial tissue from healthy to high grade tumors. Likewise, the redox ratio and FLIM analysis were able to significantly distinguish tissues characteristics. To date, only one study has used the multimodality for urothelial evaluation. Cicchi et al. have assessed TPM for healthy bladder mucosa and CIS. Their results shown a potential difference between both groups even with the redox ratio analysis. But again, the limitation to the diagnosis of CIS is a limitation for a real applicability comparatively to our study which was focused on the grade. To evaluate the redox ratio, different formula are proposed in the literature but we decided to take the most used[21], [26], [34]. Discrimination was also obtained by using the FLIM analysis. The FLIM data are bi-exponential fitted, each component fitted will be associated to one of the form of FAD in tissue, either proteinbound or free. The average amplitude lifetime translates the distribution between free and bound FAD, having longer average lifetime In tumoral tissue means that the free FAD is in higher concentration. This change in the ratio of protein-bound FAD is a consequence of the impact of the Warburg effect. In tumor, an increased metabolizing of glucose to lactate by neoplastic tissue compared with healthy 
tissue[35]. Indeed the major co-enzyme in this reaction are NADH and FAD. This is in correlation with previous results shown on healthy bladder mucosa compared to in situ carcinoma [33].

There are some limitations to discuss. First, we analyzed fixed samples. This could lead to some modifications of the fluorescence and the redox ratio analysis compared to an in-vivo analysis. Indeed, the NADH and FAD concentration decrease after the excision of the tissue and that could affect the performance of redox ratio analysis. Nevertheless, our analysis has been able to discriminate the different tissue as described in the previous literature for the redox ratio in healthy and tumoral tissues[21], [26], [34], [36]-[38]. This means that the redox ratio is also a promising tool for an invivo evaluation. The other limitation of our study is the low number of samples included. To confirm our results, we need to increase the number of samples and work on fresh biopsy analysis.

These preliminary results confirm the potential of multimodal optical analysis and represent the first step for the development of a laser fiber optical tool for peri-operative diagnosis. The possibility of discriminating the tumor's grade during the surgery is an essential tool for the oncologic management. 


\section{Acknowledgements}

This work is supported by PhysiCancer in Plan Cancer with "IMOP" grant, by CNRS with "Défi instrumental" grant, and the Institut National de Physique Nucléaire et de Physique des Particules (IN2P3). This work was done in the PIMPA Platform partly funded by the French program "Investissement d'Avenir" run by the "Agence Nationale pour la Recherche" (grant "Infrastructure d'avenir en Biologie Santé - ANR - 11-INBS-0006"). This work is supported by the French association of Urologist (AFU) with a research grant delivered to B.P. The authors declare no competing financial interests.

\section{References}

[1] R. L. Siegel, K. D. Miller, and A. Jemal, "Cancer Statistics, 2017,” CA. Cancer J. Clin., vol. 67, no. 1, pp. 7-30, Jan. 2017.

[2] M. Rouprêt et al., "European Association of Urology Guidelines on Upper Urinary Tract Urothelial Cell Carcinoma: 2015 Update," Eur. Urol., vol. 68, no. 5, pp. 868-879, Nov. 2015.

[3] M. Babjuk et al., "EAU guidelines on non-muscle-invasive urothelial carcinoma of the bladder: update 2013," Eur. Urol., vol. 64, no. 4, pp. 639-653, Oct. 2013.

[4] M. Brausi et al., "Variability in the recurrence rate at first follow-up cystoscopy after TUR in stage Ta T1 transitional cell carcinoma of the bladder: a combined analysis of seven EORTC studies," Eur. Urol., vol. 41, no. 5, pp. 523-531, May 2002.

[5] J.-N. Cornu et al., "Oncologic control obtained after exclusive flexible ureteroscopic management of upper urinary tract urothelial cell carcinoma," World J. Urol., vol. 28, no. 2, pp. 151-156, Apr. 2010.

[6] S. Daneshmand, M. L. Quek, and J. L. Huffman, "Endoscopic management of upper urinary tract transitional cell carcinoma: long-term experience," Cancer, vol. 98, no. 1, pp. 55-60, Jul. 2003.

[7] A. K. Smith et al., "Inadequacy of biopsy for diagnosis of upper tract urothelial carcinoma: implications for conservative management," Urology, vol. 78, no. 1, pp. 82-86, Jul. 2011.

[8] F. X. Keeley, D. A. Kulp, M. Bibbo, P. A. McCue, and D. H. Bagley, "Diagnostic accuracy of ureteroscopic biopsy in upper tract transitional cell carcinoma," J. Urol., vol. 157, no. 1, pp. 33-37, Jan. 1997.

[9] A. M. Batlle, "Porphyrins, porphyrias, cancer and photodynamic therapy--a model for carcinogenesis," J. Photochem. Photobiol. B, vol. 20, no. 1, pp. 5-22, Sep. 1993.

[10] K. Gono et al., "Appearance of enhanced tissue features in narrow-band endoscopic imaging,” J. Biomed. Opt., vol. 9, no. 3, pp. 568-577, Jun. 2004. 
[11] E. V. Zagaynova et al., "Contrasting properties of gold nanoparticles for optical coherence tomography: phantom, in vivo studies and Monte Carlo simulation," Phys. Med. Biol., vol. 53, no. 18, pp. 4995-5009, Sep. 2008.

[12] J.-L. Bonnal, A. Rock, A. Gagnat, S. Papadopoulos, B. Filoche, and B. Mauroy, "Confocal laser endomicroscopy of bladder tumors associated with photodynamic diagnosis: an ex vivo pilot study," Urology, vol. 80, no. 5, pp. 1162.e1-5, Nov. 2012.

[13] H. Hamzeh, C. Lefort, F. Pain, and D. Abi Haidar, "Optimization and characterization of nonlinear excitation and collection through a gradient-index lens for high-resolution nonlinear endomicroscopy," Opt. Lett., vol. 40, no. 5, p. 808, Mar. 2015.

[14] A. Ibrahim, F. Poulon, R. Habert, C. Lefort, A. Kudlinski, and D. A. Haidar, "Characterization of fiber ultrashort pulse delivery for nonlinear endomicroscopy," Opt. Express, vol. 24, no. 12, p. 12515, Jun. 2016.

[15] G. Ducourthial et al., "Development of a real-time flexible multiphoton microendoscope for label-free imaging in a live animal," Sci. Rep., vol. 5, no. 1, Nov. 2016.

[16] C. Duan, Q. Tanguy, A. Pozzi, and H. Xie, "Optical coherence tomography endoscopic probe based on a tilted MEMS mirror," Biomed. Opt. Express, vol. 7, no. 9, p. 3345, Sep. 2016.

[17] M. Zanello et al., "Multimodal optical analysis discriminates freshly extracted human sample of gliomas, metastases and meningiomas from their appropriate controls," Sci. Rep., vol. 7, p. 41724, Feb. 2017.

[18] A. Ustione and D. W. Piston, "A simple introduction to multiphoton microscopy," J. Microsc., vol. 243, no. 3, pp. 221-226, Sep. 2011.

[19] L. Marcu, "Fluorescence lifetime techniques in medical applications," Ann. Biomed. Eng., vol. 40, no. 2, pp. 304-331, Feb. 2012.

[20] A. Ibrahim et al., "Spectral and fluorescence lifetime endoscopic system using a double-clad photonic crystal fiber," Opt. Lett., vol. 41, no. 22, pp. 5214-5217, Nov. 2016.

[21] S. Palmer, K. Litvinova, E. U. Rafailov, and G. Nabi, "Detection of urinary bladder cancer cells using redox ratio and double excitation wavelengths autofluorescence," Biomed. Opt. Express, vol. 6, no. 3, pp. 977-986, Mar. 2015.

[22] C. Schäfauer et al., "Detection of bladder urothelial carcinoma using in vivo noncontact, ultraviolet excited autofluorescence measurements converted into simple color coded images: a feasibility study," J. Urol., vol. 190, no. 1, pp. 271-277, Jul. 2013.

[23] M. E. Llewellyn, R. P. J. Barretto, S. L. Delp, and M. J. Schnitzer, "Minimally invasive high-speed imaging of sarcomere contractile dynamics in mice and humans," Nature, vol. 454, no. 7205, pp. 784-788, Aug. 2008.

[24] S. M. Zhuo, J. X. Chen, T. Luo, X. S. Jiang, and S. S. Xie, "Multiphoton microscopy of unstained bladder mucosa based on two-photon excited autofluorescence and second harmonic generation," Laser Phys. Lett., vol. 6, no. 1, pp. 80-83, Jan. 2009.

[25] R. Yadav et al., "Multiphoton Microscopy of Prostate and Periprostatic Neural Tissue: A Promising Imaging Technique for Improving Nerve-Sparing Prostatectomy," J. Endourol., vol. 23, no. 5, pp. 861-867, May 2009.

[26] R. Cicchi et al., "Time- and Spectral-resolved two-photon imaging of healthy bladder mucosa and carcinoma in situ," Opt. Express, vol. 18, no. 4, pp. 3840-3849, Feb. 2010.

[27] P. A. Humphrey, H. Moch, A. L. Cubilla, T. M. Ulbright, and V. E. Reuter, "The 2016 WHO Classification of Tumours of the Urinary System and Male Genital Organs-Part B: Prostate and Bladder Tumours," Eur. Urol., vol. 70, no. 1, pp. 106-119, Jul. 2016. [28] D. A. Haidar, B. Leh, M. Zanello, and R. Siebert, "Spectral and lifetime domain measurements of rat brain tumors," Biomed. Opt. Express, vol. 6, no. 4, pp. 1219-1233, Apr. 2015.

[29] M. Zanello, A. Ibrahim, F. Poulon, P. Varlet, B. Devaux, and D. Abi Haidar, "Spectral 
and Lifetime Measurements of the Endogenous Fluorescence Variation of Freshly Resected Human Samples over Time - Measuring Endogenous Fluorescence Changes at Different Moment after Tumor or Epileptic Cortex Excision:," 2016, pp. 13-17.

[30] M. C. Skala et al., "In vivo multiphoton microscopy of NADH and FAD redox states, fluorescence lifetimes, and cellular morphology in precancerous epithelia," Proc. Natl. Acad. Sci. U. S. A., vol. 104, no. 49, pp. 19494-19499, Dec. 2007.

[31] M. C. Skala et al., "Multiphoton microscopy of endogenous fluorescence differentiates normal, precancerous, and cancerous squamous epithelial tissues," Cancer Res., vol. 65, no. 4, pp. 1180-1186, Feb. 2005.

[32] A. Varone et al., "Endogenous two-photon fluorescence imaging elucidates metabolic changes related to enhanced glycolysis and glutamine consumption in precancerous epithelial tissues," Cancer Res., vol. 74, no. 11, pp. 3067-3075, Jun. 2014.

[33] M. Jain et al., "Multiphoton microscopy: a potential intraoperative tool for the detection of carcinoma in situ in human bladder," Arch. Pathol. Lab. Med., vol. 139, no. 6, pp. 796-804, Jun. 2015.

[34] S. Palmer, K. Litvinova, A. Dunaev, J. Yubo, D. McGloin, and G. Nabi, "Optical redox ratio and endogenous porphyrins in the detection of urinary bladder cancer: A patient biopsy analysis," J. Biophotonics, vol. 10, no. 8, pp. 1062-1073, Aug. 2017.

[35] O. Warburg, F. Wind, and E. Negelein, "The metabolism of tumors in the body," J. Gen. Physiol., vol. 8, no. 6, p. 519, 1927.

[36] W. R. Zipfel, R. M. Williams, R. Christie, A. Y. Nikitin, B. T. Hyman, and W. W. Webb, "Live tissue intrinsic emission microscopy using multiphoton-excited native fluorescence and second harmonic generation," Proc. Natl. Acad. Sci. U. S. A., vol. 100, no. 12, pp. 7075-7080, Jun. 2003.

[37] L. H. Laiho, S. Pelet, T. M. Hancewicz, P. D. Kaplan, and P. T. C. So, "Two-photon 3-D mapping of ex vivo human skin endogenous fluorescence species based on fluorescence emission spectra," J. Biomed. Opt., vol. 10, no. 2, p. 024016, Apr. 2005.

[38] K. Alhallak, L. G. Rebello, T. J. Muldoon, K. P. Quinn, and N. Rajaram, "Optical redox ratio identifies metastatic potential-dependent changes in breast cancer cell metabolism," Biomed. Opt. Express, vol. 7, no. 11, pp. 4364-4374, Nov. 2016.

\section{Graphical Abstract}

In urologic oncology, quality and rapidity of diagnosis is an influent element in the choice of treatment. Intraoperative imaging techniques could help in real-time the surgeon in decision making.

This paper presents a new technology for intraoperative assessment of the nature of bladder tissue. A multimodal tools with two-photon exciton and quantitative-qualitative detection. Histological landmarks were found in two-photon images of biopsy and quantitative measurements gave numerical indicators of malignancy. 


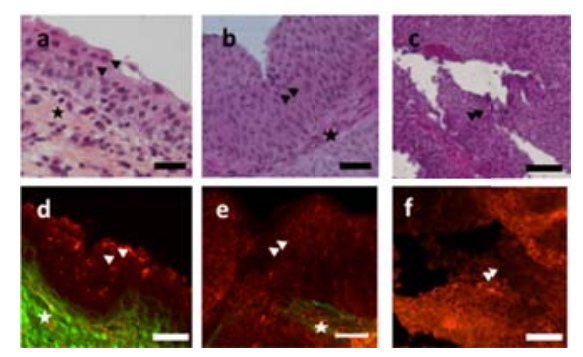

